\title{
Conocimientos, Actitudes y Prácticas que posee la población universitaria de la UEES en relación a la Salud Bucal. Estudio Piloto realizado en el 2005
}

\author{
Knowledge, Attitude and Practice that the university \\ population of the UEES has in relation to Dental Health. \\ Siudy Pilot 2005
}

Pérez Oljva, Elizabeth

Dactora en Cinugia Dental UEES 1994

Master en Administración y Docencia Unizersitaria UEES 2001 Investigadora de la Direción de Intestigación de la UEES. E-mail: elizabetholiva2002@gmail.com

Rivera de Villacorta, Brenda Lissette

Doctora en Cirugia Dental UEES 2002. Experto en Alención Odontologica a paciente especial, Universidad de Complutense de Madrid, España 2003. Profesor Titular del Departamento de Odontologia Preventiva, área de Odontopediatría de la UEES. E-mail: villarivera2002@hotmail.com

Resumen

$D$ iagnosticar los Conocimientos, Actitudes y Prácticas ue posee este sector de la población salvadoreña permitirá proponer acciones preventivas y curativas referentes a la Salud Bucal y acordes a la realidad que vize esta población, pero también evidenciará la importancia que le brindan los Saluadoreños a la Salud Bucal con el propóxito de reorientar la formación de los futuros odontólogos con una visión preventiza. Este estudio considerado como piloto, aporta información relevante de este sector de la población universilaria de la UEES, ya que el conocimiento del estado de salud bucal proponciona una base aderuada para estimar las necesidades actuales y de futuras asistencias odontológicas de la misma. Se pretende que la segunda fase de este estudio se realice a nivel nacional. Al finalizar el estudio se concluye que en cuanto a conocimientos los estudiantes conocen sobre los hábitos de higiene bucal y sobre las recomendaciones de la visila al menos una vez al año al odontólogo. La mavoría de ellos conocen alounas consecuencias relacionadas a la mala salud bucal y de las repercusiones en la salud general. En cuanto a actitudes, para la mayoria de los estudiantes es buena, por lo lanto es necesario desarrollar actividades en ellos que les ayude a influir en pro de modos y estilos de vida favorables, de manera que éstas ayuden a rescatar una correcta higiene dental. En relación a la Práctica la mayoria de los estudiantes al utilizar los diferentes aditamentos de higiene bucal se autocalifican con mejores hábilos de higiene bucal.

Palabras clazes: Conocimientos, Actitudes, Prácticas, Salud Bucal, Población Universitaria, Prevención.
Abstract

M aking a diagnose of the knowledge, attitude and practice this sector of the Saluadorian population has, will permit to propose preventive and curative actions referring to the Dental Health and agreed to the reality that this population lives, but also will demostrate the importance that the Salvadoreans offer to their Dental Health in order to rearient the training of future dentists with a preventive vision. This study is considered as a pilot study because has been made among the student population of the UEES with the purpose of validating the interviezering guide and obtaining data referring to this population. We intend that the second phase of this study would be made nationzerde. When the study ends, it is concluded that concerning learning, the students. know about the habits of dental hygiene and the recommendations of visiting the dentist at least once a year. Most of them knowe some consequences related to the bad dental heatth. Regarding attitude, for most of the students it is not good, although they have knowetedge about the consequences of bad dental health and of the repercussions in the general health and as for Practice, most of the students when use the different additions from dental hygiene are described as beller higiene habits.

Key Words: Knowoledge, Attitudes, Practices, Oral Health, Population University, Prevention. 


\section{Introducción}

Desde tiempos inmemoriales se sabe que los conocimientos y prácticas en relación con la salud son muy importantes para la prevención y curación de las enfermedades. Sin embargo, la caries dental y la enfermedad periodontal aparecen entre las primeras causas de morbilidad bucal a nivel mundial y El Salvador no está exento de estos problemas, lastimosamente, estos no son temas trascendentales para la población salvadoreña debido a la crisis económica que atraviesa nuestro país. La mayoría de los Salvadoreños no se preocupan por asistir al odontólogo a menos de que tenga un problema tan grande y doloroso que le incapacite a seguir con la rutina diaria, ya que del ingreso económico que pudiera tener primero opta por cubrir las necesidades básicas de comida, vivienda y si alcanza educación.

Diagnosticar los Conocimientos, Actitudes y Prácticas que posee la población salvadoreña permitirá proponer acciones preventivas y curativas referentes a la Salud Bucal y acordes a la realidad que vive la población, además evidenciará la importancia que le brindan los Salvadoreños a la Salud Bucal con el propósito de reorientar la formación de los futuros odontólogos con una visión preventiva. Este estudio considerado como piloto, aporta información relevante de este sector de la población universitaria de la UEES, ya que el conocimiento del estado de salud bucal proporciona una base adecuada para estimar las necesidades actuales y de futuras asistencias odontológicas de la misma. Se pretende que la segunda fase de este estudio se realice a nivel nacional.

La Organización Mundial de la Salud (OMS) en el 2003 publicó un nuevo informe sobre el problema Mundial de las enfermedades bucodentales, como la caries dental, la periodontitis (enfermedad gingival) y los cánceres de la boca y la faringe son un problema de salud de alcance mundial que afecta a los países industrializados y, cada vez con mayor frecuencia, a los paises en desarrollo, haciendo referencia en especial entre las comunidades más pobres, la OMS ha declarado que se estima que cinco mil millones de personas en el planeta han sufrido caries dental. Según la OMS los efectos de las enfermedades bucodentales en términos de dolor, sufrimiento, deterioro funcional y disminución de la calidad de vida son considerables y costosos. Se estima que el tratamiento representa entre el $5 \%$ y el $10 \%$ del gasto sanitario de los países industrializados, y está por encima de los recursos de muchos países en desarrollo. En El Salvador actualmente los padecimientos bucales y de los dientes no son temas trascendentales, sobre todo para la población salvadoreña menos favorecida, la que en estos momentos atraviesa una grave crisis económica. La mayoría de las salvadoreños opta por gastar lo que les ingresa en cubrir algunas necesidades básicas siendo estas la de alimentación o de salud, en la medida de que no hay dinero para satisfacer estas necesidades, al no alcanzar siquiera el salario mínimo para cubrir la canasta básica la gran mayoría de la población no se está nutriendo. Esto es aun más grave cuando en esta población se incluyen las mujeres embarazadas y los niños, lo cual numerosos estudios recientemente han demostrado y lo reafirma García (2004) que si durante el embarazo no se ingieren suficientes proteinas la calidad de los dientes de los niños es menor y puede afectarse además la forma de los mismos, en ese mismo articulo se hace referencia a las repercusiones en cuanto al desarrollo cerebral que también se produce el cual es insuficiente e irreversible.

La malnutrición puede retardar la erupción y composición de los dientes primarios, además del hucso de sostén de éstos y en consecuencia aumentar la prevalencia de caries. García afirma que quienes desde el vientre materno no reciben la suficiente nutrición tendrán más problemas bucodentarios, que el resto de la población. Si durante la etapa de formación el feto no tuvo proteinas suficientes para que su cerebro se desarrollara a cabalidad, lucgo de nacido pueden darle todas las proteínas posibles, pero ya será tarde y no se alcanzará el completo desarrollo. Otros estudios demuestran la relación entre enfermedades de la boca y enfermedades sistémicas. Romero Diaz reafirma lo antes mencionado en su articulo "Enfermedades bucales con repercusión sistémica" en donde relaciona la enfermedad periodontal con las enfermedades cardiovasculares.

García (2004) argumenta que la sociedad, en alguna forma, limita el futuro de una cantidad de seres humanos porque, simplemente, el cerebro no les rendirá con capacidad normal. Eso es grave, pero en lo relacionado al campo de la odontología, es vital cl problema de las caries. Así mismo hace referencia de un informe del doctor Saccher, el cual señala que otro obstáculo en la búsqueda y obtención de ayuda profesional es la falta de conocimiento de la población general sobre la importancia de la salud oral. La pregunta constante es sobre quien sería el responsable de esta realidad y la responsabilidad recae en los odontólogos porque no se han ocupado de educar a la comunidad, sino de hacer restauraciones bonitas, de prepararse en el aspecto científico únicamente y se han olvidado del aspecto humano en relación a la enseñanza de la higiene y prevención de la enfermedad dental en el consultorio.

En la actualidad el gremio odontológico y las escuelas formadoras de odontólogos realizan algunos esfuerzos que llevan a concientizar a los futuros odontólogos sobre la necesidad de educar a la población Salvadoreña acerca de la importancia de mantener una buena Salud bucal lo cual repercute en el resto de los órganos del cuerpo.

En nuestro país se han obtenido algunos logros en el campo de la salud bucal, existe un estudio epidemiológico sobre la caries y la fluorosis dental, esta investigación realizada por el Ministerio de Salud Publica y Asistencia Social (MSPAS) en el 2002, refiere que la prevalencia de caries encontrada en 2000 escolares fue del $61 \%$. El $28.2 \%$ de los escolares 
de 6 años poseen dentadura sana; el promedio CPOD ${ }^{\prime}$ en los niños y niñas de 12 años es de 1.3 piezas dentales afectadas, ascendiendo hasta un promedio de 2.4 para la edad 15 años. En la evaluación de los índices de CPOD y $\operatorname{ceod}^{2}$, la Caries tuvo mayor prevalencia en relación a los otros componentes Perdidos y Obturados que conforman dichos índices. En ese mismo estudio el $80.45 \%$ de los sujetos de estudio presentaron algún nivel de placa bacteriana, además se observó en el $20.6 \%$ de los sujetos la necesidad de tratamiento inmediato debido a caries profunda.

Otros provectos o investigaciones referentes al árca de salud bucal, tales como, estudios sobre la prevalencia de lesiones cariosas, memorias de actividades realizadas en el servicio social, evaluación de estado de salud bucal de un grupo poblacional, estadística periodontal de pacientes atendidos en las clínicas, etc. que abordan parte de la población, han sido tomados como base para considerar de suma importancia la realización del presente estudio, ya que al diagnosticar los Conocimientos, Actitudes y Practicas (CAP's) de los estudiantes de la UEES en relación a la salud bucal es factible proponer programas preventivos de atención bucal acordes a la realidad de la población, ya que de esta manera, se determinaran los conocimientos, medidas preventivas empleadas por este grupo de estudio y se identificarán los problemas y tratamientos bucales que estos presentan.

Así mismo, compañías como Colgate Palmolive y Oral B, realizan esfuerzos aislados por apoyar iniciativas de educación en la prevención de enfermedades bucales a grupos específicos de salvadoreños, tales como la creación de centros o zonas de prevención en las Facultades de Odontología de algunas Universidades, sin embargo, no todos los salvadoreños tienen acceso a estas iniciativas. Es importante mencionar que estas compañias poseen algunos programas para educar e informar a la población en general, sin embargo, son esfuerzos que aún no logran provocar un impacto pues no se les da seguimiento.

Otero y Toledo en un estudio realizado en el 2006 , mencionan que los estudios epidemiológicos bucodentales responden fundamentalmente a un interés por el conocimiento del estado de salud oral, para proceder a instauración de programas de intervención y prevención, entre otros. Si se retoma la frase de Avendaño "La base de la salud bucal es la Prevención e Higiene", la higiene bucal como tal, es un factor determinante para el mantenimiento de una salud bucal aceptable, en este sentido, las condiciones de la población con respecto a esta conducta, amerita de una educación orientada hacia la adaptación de patrones que favorezcan la higiene bucal.
Por otro lado, es importante considerar que a nivel nacional las entidades de salud están enfocando la práctica odontológica a una de filosofía de trabajo preventivo, el estado de salud bucal que presenta la población salvadoreña debería ser un tema de interés para todos los odontólogos, sobre todo aquellos que se encuentran en proceso de formación en las aulas universitarias.

\section{Metodología de la investigación}

En este estudio de corte transeccional descriptivo, la muestra sistemática fue de 345 estudiantes, seleccionada aleatoriamente con un error del $5 \%$, de una población total de 3,246. Sin embargo, al momento de buscar a los estudiantes estos no se encontraban en las aulas en los horarios que se habían proporcionado, por lo que la muestra final con la que se trabajó fue de 257 estudiantes.

Los participantes del estudio fueron estudiantes de la UEES que oscilan entre las edades de 15 a 60 años pertenecientes a las diferentes facultades de la universidad, excepto los estudiantes de la facultad de odontología.

El Instrumento utilizado fue una guía de entrevista estructurada con 47 reactivos. Las dimensiones referidas a conocimientos, hábitos y prácticas de salud bucal se investigan con preguntas abiertas y cerradas. La dimensión de actitudes se identifica por medio de una escala de Likert con cinco valoraciones de respuestas.

Para este estudio se entenderá como Conocimiento al conjunto de datos que se tiene respecto a los hábitos de higiene y salud bucal; Actitud como la manera o expresión de los estudiantes para estar dispuesto a comportarse u obrar en relación a la salud bucal y la Práctica como la aplicación de conocimientos teóricos brindados por una persona sobre salud bucal y que se realizan de manera habitual o por costumbre.

La validación del Instrumento fuc realizado tanto en contenido como en formato y estilo. La validación de contenido fue realizada por dos expertos, quienes revisaron el borrador del instrumento para garantizar la claridad y pertinencia del contenido, en tanto que tres expertos en investigación social examinaron formato y estilo.

Para la recolección de los datos participaron tres estudiantes en servicio social quienes fueron previamente capacitados para llevar a cabo la encuesta por entrevista personal, en donde los encuestadores, explicaron a los participantes el objetivo de la investigación y se entrevistaron a los que aceptaron y estuvieron dispuestos a contestar.

Se realizó una codificación del instrumento para elaborar la base de datos en el programa SPSS versión

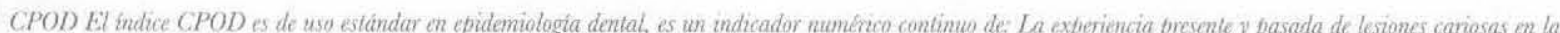
corona de los dientes permanentes (componenie C y dientes cariados), y la experiencia de secuelas de la caries coronal, ya sean dientes perdidas por caries fomponente $P$ o dientes perdidos) o dientes obturados $/$ restaurados por medio de procedimientos protésicos dentales (componentes $O$ o dientes obturados).

${ }^{2}$ ceod indice de epidemialogia dental apticado a los dientes primarios, deriduos, suredáneas o de leche.
} 
11.0 para Windows e ingresarlas al sistema, generando medidas de tendencia central, tablas de frecuencia y otras medidas de proporción para la interpretación de datos y elaboración de las respectivas conclusiones y recomendaciones.

\section{Resultados y Discusión}

Se encuestaron un total de 257 estudiantes. El 33\% pertenecen a la facultad de Medicina, le sigue con el $23 \%$ los de Ciencias Sociales, el $18 \%$ a Ciencias Jurídicas e Ingenierías respectivamente y el $8 \%$ a Empresariales. Respecto al nivel de estudio que poseían al momento de la encuesta, el $48 \%$ se ubicaban entre el primer y quinto ciclo, el $52 \%$ restante se ubican entre el sexto y décimo ciclo de estudios de sus respectivas carreras. El $53 \%$ de la población encuestada oscila entre los 20 y 23 años, y el $52 \%$ pertenecen al sexo femenino.

\section{A. Hábitos de higiene bucal.}

El gráfico 1 muestra que el $65 \%$ de los encuestados refieren tener buenos hábitos de higiene bucal, seguido del $19 \%$ que son excelentes, el $14 \%$ regulares y el $2 \%$ malos. A diferencia de un estudio realizado por Suárez y Pombo en el 2003 quienes encontraron que un $58.6 \%$ resultaron con una evaluación de regular. En un estudio realizado por Brenes y Sosa en 1986 en una población de adolescentes costarricenses registraron el índice de higiene oral como excelente, ya que en él se estableció un Índice basado en la presencia clínica de placa bacteriana.

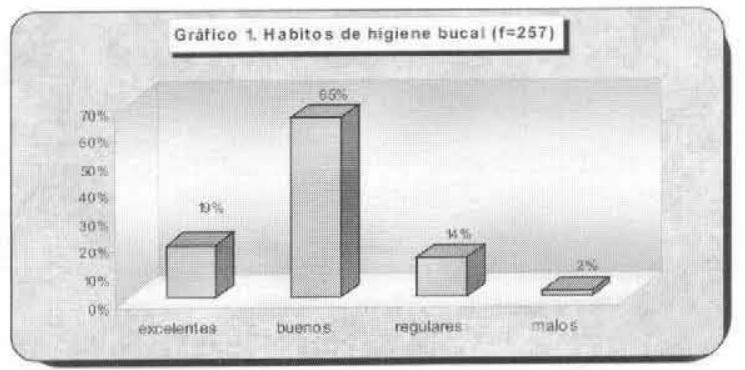

\section{Cepillado y Cepillo dental.}

En relación al cepillado, el 98\% de los encuestados refiere cepillarse los dientes lo que estaría indicando que al menos tienen la conducta de cepillarse los dientes. Este dato coincide por lo encontrado por Sosa y col. (2003) en un estudio realizado en una población escolar de Venezuela 7 a 13 años de una institución educativa.

De los que se cepillan el $78 \%$ de 254 estudiantes encuestados manifiestan cepillarse tres o más veces al día, el $18 \%$ dos veces y el $4 \%$ tan solo se cepilla una vez al día. Esto difiere de lo encontrado por Brenes y Sosa (1986) y Sosa (2003), en ambos estudios, la frecuencia de cepillado fue mayor de una a dos veces al día, esto podría explicarse mejor considerando que las personas que participaron en ambos estudios son jóvenes de menor edad y de un nivel educativo básico. Otero y Toledo en el 2006 encontraron que uno de los aspectos que más encontraron dificultad en la población que ellos estudiaron es en la frecuencia de cepillado.

En relación a la marca de cepillo dental que utilizan de 240 encuestados, el $49 \%$ respondió utilizar la marca Oral B, el $44 \%$ Colgate y un $5 \%$ Gum. Respecto al tipo de cerdas que tiene el cepillo que utilizan los encuestados, el $52 \%$ utiliza cepillo con cerdas medianas, el $42 \%$ con cerdas suaves y un $6 \%$ manifestó tener cepillos con cerdas duras. En cuanto a los aspectos que consideran los encucstados al comprar un cepillo dental, el $60 \%$ manifiesta que se fijan en la marca, cl $25 \%$ en que sea la que indicó el dentista y el 15\% en el precio.

\section{Pasta dental.}

El $98 \%$ de los encuestados manifiestan utilizar pasta dental para cepillarse los dientes. Respecto a la marca de la pasta dental, de 218 el $79 \%$ utiliza Colgate, el 9\% Close-up, el 7\% Crest y el 4\% Aquafresh. El $26 \%$ de los encuestados al comprar una pasta dental se fijan en que blanquee los dientes, el $25 \%$ que contenga flúor, el $21 \%$ en la marca, el $17 \%$ en que sea la que indicó el dentista y el $11 \%$ en el precio.

\section{Seda dental.}

El $49 \%$ de los encuestados respondió que si usa la seda dental para el aseo de sus dientes, mientras que el $28 \%$ respondió que no y el $23 \%$ que a veces. De 97 encuestados el $47 \%$ utilizan la marca Colgate y el $46 \%$ utiliza la marca Oral B. El 7\% respondió utilizar otras marcas entre las que mencionan Jhonson, Gum y Perriot

Respecto a la frecuencia del uso de la seda dental en cl día para 183 encuestados es que el $36 \%$ la utiliza una vez, el $32 \%$ la usa dos veces, el $19 \%$ tres o mas veces y el $13 \%$ solo cuando siente que se le traba. En relación a la persona que le indicó utilizar seda dental, el $69 \%$ respondió que fue el dentista, el $25 \%$ los padres y un $5 \%$ respondieron los profesores. Un $1 \%$ respondió que otras personas y hacen referencia a otros familiares y amigos.

En una nota publicada por EUROPA PRESS afirman que sólo el $10 \%$ de la población usa correctamente seda dental en su higiene bucodental, según los datos de un análisis sobre hábitos de higiene dental en españoles y portugueses. Este estudio ha sido realizado por la Sociedad Española de Periodoncia, la Sociedad Portuguesa de Periodontología e Implantes y la Sociedad Española de Epidemiología y Salud Pública Oral. Con lo anterior puede observarse, entonces que el uso de la seda dental no es una práctica muy difundida, lo que indica que es importante que el odontólogo intensifique las acciones de promover el uso del mismo. 


\section{Enjuague Bucal}

Respecto al enjuague el $62 \%$ afirmó utilizarlo, el $20 \%$ respondió no utilizar enjuague y el $18 \%$ respondió que a veces lo utiliza. En rclación al número de veces al día que utilizan el enjuague bucal, el 37\% que lo utilizan tres o más veces al día, el $32 \%$ una vez al día y un $31 \%$ lo utilizan dos veces al día. El $93 \%$ manifiestan utilizarlo después del cepillado y el $7 \%$ antes del cepillado. De 139 estudiantes que respondieron sobre las marcas de enjuague bucal, se obtuvo que el $50 \%$ utiliza Listerine, el $36 \%$ Oral B, el $6 \%$ Colgate (Plax) el 3\% utilizan Scope, el 2\% Fluor King y el $3 \%$ otros. Entre otros enjuagues mencionan Corsident, Crest, Lacer Ortodent, Medident. Respecto a la persona que le indicó utilizar enjuague bucal, el $59^{\circ} \%$ respondió que fue el dentista, el $38 \%$ manifestó que los padres y el $3 \%$ respondicron que los profesores.

Puede observarse el papel preponderante que el Odontólogo posee como ente orientador en los aspectos preventivos de la salud bucal.

\section{B. Relación de los hábitos de higiene bucal con otras variables}

En este apartado se va a considerar la variable higiene bucal relacionándola con facultad a la que pertenecen, edad, sexo, cepillado dental, uso de cepillo, seda dental y uso de enjuague bucal, por lo que se irá realizando una descripción de cada una de cllas relacionándola con los hábitos de higiene bucal.

\section{Relación Hábitos de higiene bucal y Facultad}

$\mathrm{Al}$ relacionar la facultad a la que pertenecen los encuestados con los hábitos de higiene bucal la mayoría de los estudiantes de las facultades encuestadas respondieron tener buenos hábitos. Lis necesario aclarar que para el estudio se excluyeron a los estudiantes de la facultad de odontología. Los estudiantes de la facultad de Medicina y Ciencias Sociales le siguen con mayor porcentaje respecto a tencr excelentes hábitos. En alguna medida esto podría tener la explicación de que los de Medicina al ser del árca de la salud, dentro de su formación debe estar contemplado la prevención de todo tipo de enfermedades. Los de Ciencias Sociales al estar inmersos en una formación del área educativa probablemente se les instruya en el cuido en general de su cuerpo. Esto fue confirmado con la prueba de prucba de chi cuadrado con un $\alpha 0.05$, en donde se observan mejores hábitos de higiene bucal en los estudiantes que pertenecen a las facultades referidas a educación y salud. consecuentemente, los hábitos de higiene bucal no son tan buenos en los estudiantes que pertenecen a las facultades de las otras áreas del conocimiento.

En cste sentido se coincide con lo afirmado por Armenteros, Hernández y Olalde en que es necesario modificar la conducta individual de cada persona encaminadas a la transmisión de conocimientos sobre la importancia de la salud bucal con el fin de promover un cambio de actitud la cual será reflejada en la conducta que éste tenga sobre los hábitos de higiene bucal.

\section{Relación entre la Edad y Hábitos}

Al relacionar la edad con los hábitos de higiene bucal de los encuestados la mayoría de los estudiantes entre los 20 y 23 años refieren tener buenos hábitos. $\mathrm{Al}$ plantearse la Ho respecto a que la edad y los hábitos de higiene bucal son independientes y realizar la prueba de chi cuadrado con un $\alpha 0.05$, se establece una dependencia o fucrte relación entre ambas variables, esto significa que a menor edad de los encuestados se observan mejores hábitos de higiene bucal y a mavor edad los hábitos de higiene bucal no son tan buenos. Esto coincide con el estudio realizado por Suárez y Pombo en el 2003 quienes encontraron que el $58.6 \%$ presentaron una evaluación de regular, con las mayores cifras en los grupos de 35-59. Tambien cn un artículo publicado en ELMUNDOSALUD.COM titulado "Los españoles se cepillan los dientes poco y mal" se menciona que los que menos se preocupan de su higiene oral son las personas mayores de 65 años, ya que un $14,6 \%$ de este segmento de población reconoce no cepillarsc nunca.

\section{Relación entre el sexo de los encuestados} y los Hábitos de Higiene Bucal

La mayoría de los que refieren tener buenos hábitos de higiene bucal pertenecen al sexo femenino. Sin embargo, se observa una leve diferencia respecto a los que refieren tener excclentes y regulares hábitos en donde la mayoría corresponden al sexo masculino. Esto coincide por lo encontrado por Brenes y Sosa (1986) en su estudio sobre epidemiología y accesibilidad a los servicios odontológicos de adolescentes en donde encontró que los adolescentes masculinos muestran un nivel de higiene más favorable que los hallados en el sexo femenino.

Para ver si existe alguna relación entre ambas variables se sometió a prueba estadística de chi cuadrado con un $\alpha 0.05$, la hipótesis nula que dice: "no existe relación entre el sexo al que pertenecen los encuestados y los hábitos de higiene bucal manifestados", estableciéndose una dependencia o fuerte relación entre ambas variables, esto significa que las personas de sexo femenino tienden a tener mejores hábitos de higiene bucal que los del sexo masculino.

\section{Relación entre los Hábitos de Higiene Bucal y el Cepillado Dental}

$\mathrm{Al}$ relacionar las respuestas de los que respondieron cepillarse los dientes con los hábitos de higiene bucal se observa que todos los estudiantes que se cepillan son los que respondieron tener buenos hábitos. Así mismo se obtuvo que en los grupos que respondieron tener hábitos de higienc bucal regulares y malos están los que no se cepillan. Sin embargo, se observó que del grupo que respondieron tener excelentes hábitos 
hubo una persona que manifestó no cepillarse. $\mathrm{Al}$ aplicar la prueba de chi cuadrado con un a $\propto 0.05$ se concluye que existe una relación entre ambas variables por consiguiente los hábitos de higiene bucal serán mejores en la medida en que se realicc el cepillado dental y viceversa.

\section{Relación entre el número de veces que} realiza el Cepillado Dental y Hábitos Al relacionar las variables número de veces que se cepilla al día con los hábitos de higiene bucal se obtuvo que entre más aumenta el número de veces del cepillado en el día los hábitos de higiene bucal son mejores. Esto denota que los encuestados están conscientes de que entre menos se cepillen, sus hábitos de higiene bucal no serán tan buenos. Al realizar la prueba de chi cuadrado con un $\alpha 0.05$ se reafirma que el número de veces en que se cepille una persona está fuertemente relacionada con mejores hábitos de higiene bucal manifestados por la misma.

\section{Relación entre los Hábitos de higiene bucal y el uso de pasta dental.}

$\mathrm{Al}$ relacionar las respuestas de los que respondieron usar pasta dental al cepillarse con los hábitos de higiene bucal se observa que todos los estudiantes que usan pasta dental para cepillarse son los que respondieron tener entre excelentes y buenos hábitos. Así mismo se observa que en los grupos que respondieron tener hábitos de higiene bucal regulares y malos están los que respondieron no utilizar pasta dental para cepillarse.

Al realizar la prueba estadística de chi cuadrado con un $\alpha 0.05$, se establece una dependencia o fuerte relación entre ambas variables, esto significa que las personas que usan pasta dental tienden a tener mejores hábitos de higiene bucal que los que nos los usan.

\section{Relación entre el uso de la seda dental y Hábitos}

Al relacionar el uso de la seda dental con los hábitos de higiene bucal se obtuvo que el mayor porcentaje de los que respondicron usar la seda dental están ubicados entre los que tienen buenos o excelentes hábitos de higiene bucal. $\mathrm{Al}$ realizar la prueba de chi cuadrado con un $\alpha 0.05$ se reafirma que la variable uso de la seda dental está fuertemente relacionada con los hábitos de higiene bucal que poseen, por lo tanto se concluye que existe relación entre los hábitos de higiene bucal manifestados y el uso de seda dental.

\section{Relación entre número de veces que usa la seda dental y Hábitos}

$\mathrm{Al}$ relacionar esta variable con los hábitos de higiene bucal se observa que el mayor porcentaje de los que respondieron usar la seda dental de una a más veces en el día, están ubicados entre los que tienen buenos o excelentes hábitos de higiene. Sin embargo, llama la atención que algunos encuestados que respondieron utilizar la seda dental solo cuando sienten que se les traba la comida, consideran tener buenos o excelentes hábitos, lo que evidencia la falta de concientización respecto a utilizar la seda un mayor número de veces al día. $\mathrm{Al}$ realizar la prueba de chi cuadrado con un $\alpha 0.05$ se reafirma, que el número de veces que utiliza la seda dental está fuertemente relacionada con los hábitos de higiene bucal que poseen.

\section{Relación entre el enjuague bucal y Hábitos}

Al relacionar cl uso de enjuague bucal con los hábitos de higiene bucal se obtuvo que el mayor porcentaje de los que respondieron usar enjuague bucal estén ubicados entre los que tienen excelentes, buenos e incluso regulares hábitos de higiene bucal. Al realizar la prueba de chi cuadrado con un $\alpha 0.05$ se reafirma que la variable uso de enjuague bucal está fuertemente relacionada con los hábitos de higiene bucal que poseen, por lo tanto, se concluye que existe relación entre los hábitos de higiene bucal manifestados y el uso de enjuague bucal.

\section{Relación entre número de veces que usa el enjuague bucal y Hábitos.}

$\mathrm{Al}$ relacionar esta variable con los hábitos de higiene bucal se obtuvo que el mayor porcentaje de los que respondicron usar enjuague bucal de dos a tres veces en cl día están ubicados entre los que tienen buenos hábitos de higiene. Sin embargo, es curioso observar que aún los que utilizan una vez al día el enjuague consideran tener buenos hábitos, indicando que no existe concientización o no le dan importancia al uso del mismo. Es importante tambien hacer notar, que los que consideran tener excelentes hábitos están los que utilizan el enjuague tres veces al día y en menor porcentaje los que lo utilizan de una a dos veces al día. $\mathrm{Al}$ realizar la prueba de chi cuadrado $\operatorname{con} u n \alpha$. 0.05 se reafirma, que el número de veces que utiliza el enjuague bucal está fuertemente relacionada con los hábitos de higiene que refieren.

\section{Problemas y tratamientos dentales.}

En este apartado se consideraran los problemas dentales referidos por los encuestados y los tratamientos realizados, en donde se muestran los tratamientos realizados y el personal que los ha realizado. Así mismo se verán algunos resultados respecto al Odontólogo tales como el número de veces que visita al dentista, si acude siempre al mismo dentista, las razones por las que acude al dentista, calificación a las tarifas que cobran los odontólogos, confianza que le merecen los dentistas de nuestro país y a extranjeros, aspectos en los que se basan para elegir a su odontólogo y las razones por las que lo recomienda.

\section{Problemas bucales referidos.}

El 35\% de los 257 encuestados no refieren tener algún problema bucal, mientras que el $65 \%$ restante si refieren tener problemas de caries, sensibilidad dental y dolor dental, le siguen otros problemas estéticos tales como dientes amontonados y manchas en los dientes, otros manifiestan tener problemas de sangramiento de las encías, mal aliento, mal sabor en la boca, abscesos o postemas y llagas o úlceras en la boca. 


\section{Tratamientos realizados.}

E1 $86 \%$ de los encuestados refieren habérseles realizado algún tratamiento dental en su boca, el $12 \%$ respondieron que no y el $2 \%$ no respondió. Puede observarse que la mayoría ha recibido algún tipo de tratamiento, esto coincide con lo encontrado por Brenes y Sosa (1986) en el estudio sobre epidemiología bucal y accesibilidad de un grupo de adolescentes realizado en Costa Rica, en donde, el $95 \%$ de la población estudiada había recibido algún tipo de tratamiento en alguna etapa de su vida. El $79 \%$ de los encuestados respondieron que los tratamientos fueron realizados por el odontólogo, el $2 \%$ por el técnico dental y el $2 \%$ por estudiantes de odontología.

\section{Visitas al Odontólogo.}

El 28\% de los encuestados visitan dos veces al año al dentista, seguido del $23 \%$ que lo visita solo una vez al año. El 33\% de los encuestados coinciden en las respuestas en donde manifiestan visitarlo tres veces al año, más de cuatro veces y nunca lo visita $(11 \%$ respectivamente), el $4 \%$ respondió que visita cuatro veces al año. Presumiblemente las frecuentes visitas (de 3 o mas de 4 veces) se deban a que los participantes están bajo tratamiento de ortodoncia, lo que necesariamente les obligue a dicha asistencia. Estos resultados difieren de lo encontrado por Brenes y Sosa, en donde el $72 \%$ refieren visitar al odontólogo una vez al año, sin identificar el motivo por el cual asisten y el $24 \%$ asisten dos veces al año. El $75 \%$ de lo encuestados respondieron visitar siempre al mismo dentista, mientras que el $21 \%$ respondió que no. $\mathrm{Al}$ preguntarles las razones por las que acude al dentista el $44 \%$ respondió que por chequeos rutinarios, el $19 \%$ solo lo visita cuando le molesta algún diente, el $25 \%$ dijo que por salud y un $9 \%$ solo por emergencia.

En relación a las tarifas que cobran los odontólogos el $37 \%$ de los encuestados las califican de caras, el $36 \%$ manifiestan que están adecuadas, el $21 \%$ las consideran muy caras, el $2 \%$ las consideran entre económicas y muy económicas, $1 \%$ respectivamente.

$\mathrm{Al}$ comparar las respuestas respecto a la confianza que le merecen los odontólogos de nuestro país y los extranjeros. El $41 \%$ de los encuestados refieren tener bastante confianza en los nacionales mientras que solo un $33 \%$ refieren tener bastante confianza en los extranjeros. El 37\% refiere mucha confianza en los nacionales mientras que en los extranjeros solo el $29 \%$. Esto significa que la población encuestada prefiere en su mayoría que los tratamientos sean realizados por los odontólogos graduados de las diferentes escuelas del país.

El gráfico 2 muestra que entre los aspectos en los que se basan los encuestados para elegir a su dentista están con un $69 \%$ la confianza le sigue las referencias
Gráfico 2. Aspectos en los que se bas an los encuestados para elegir a su dentista

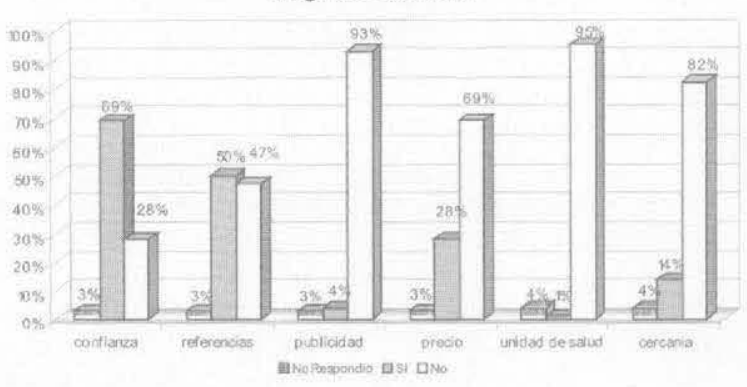

de éste con un $50 \%$ y los precios con un $28 \%$. También se aprecia que el $95 \%$ de los encuestados no considera que el dentista trabaje en una unidad de salud para elegirlo, el $93 \%$ no considera la publicidad que este haga, el $82 \%$ no considera la cercanía y el $69 \%$ tampoco considera los precios.

El gráfico 3 muestra que las razones por las que los encuestados recomiendan a un dentista, así se tiene que el $73 \%$ lo recomiendan por la calidad de los trabajos, el $38 \%$ por el trato que recibe, el $36 \%$ por la tarifa que cobra y el $34 \%$ por la experiencia que éste tiene. En menores porcentajes se observa que es por la universidad de donde se graduó y la edad del dentista, $4 \%$ y $2 \%$ respectivamente. La gráfica también muestra que el $93 \%$ de los encuestados no recomiendan a un dentista por la edad que este tenga, el $90 \%$ ni por la universidad de donde se graduó, le siguen la experiencia, la tarifa ni el trato con un $60 \%$, $59 \%$ y $57 \%$ respectivamente.

Gráfico 3. Razones por las que recomienda a un dentista

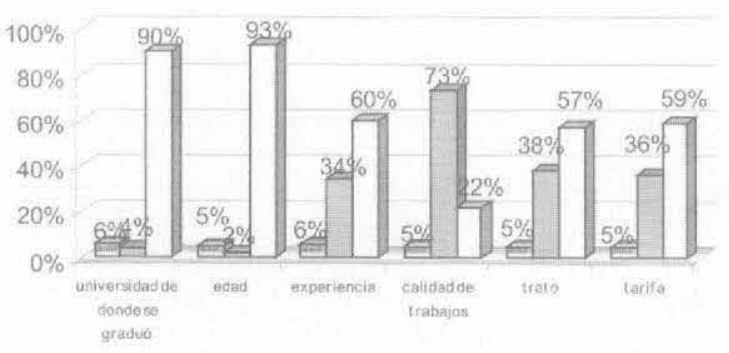

国o Raspondió $\square$ Si $\square$ No

\section{Actitudes hacia la prevención dental.}

8 de cada 10 encuestados están consientes que el costo de los tratamientos preventivos es más barato que el tratamiento específico de la enfermedad. 7 de cada 10 encuestados concuerdan que el cepillado dental no es suficiente como para no ir periódicamente al dentista. Sin embargo, 2 de cada 10 opinan que es suficiente. 
9 de cada 10 de los encuestados están entre totalmente de acuerdo y de acuerdo en que los odontólogos deben interesarse por informar a las personas sobre como mantener una buena higiene bucal. 7 de cada 10 encuestados concucrdan en que el mantenimiento de la salud bucal contribuye a mantener un buen estado de salud gencral. 1 de cada 10 está en desacuerdo.

7 de cada 10 de los encuestados concuerdan respecto a que las enfermedades bucales alteran la salud general de las personas. 1 de cada 10 considera que no hay relación.

9 de cada 10 de los encuestados coinciden en que al conocer el estado en el que se encuentra la boca les motiva a buscar tratamientos dentales. 9 de cada 10 de los encuestados concuerdan en que el trato que el odontólogo le da al paciente es vital para continuar un tratamiento dental.

9 de cada 10 de los encuestados coinciden en que la forma en la que se cepillan los dientes influye en la correcta limpieza de los dientes.

6 de cada 10 de los encuestados están entre totalmente en desacuerdo y en desacuerdo respecto a la afirmación "el cepillado de los dientes es suficiente para eliminar la caries"

7 de cada 10 de los encuestados están entre totalmente en desacuerdo y en desacuerdo respecto a la afirmación "se debe acudir al dentista sólo cuando se tiene un problema en la boca" mientras que 2 de cada 10 están de acuerdo y totalmente de acuerdo.

\section{Conclusiones}

En cuanto a conocimientos se concluye que los estudiantes conocen sobre los hábitos de higiene bucal y sobre las recomendaciones de la visita al menos una vez al año al odontólogo. La mavoría de ellos conocen algunas consecuencias relacionadas a la mala salud bucal y de las repercusiones en la salud general.

En cuanto a actitudes se concluye que para la mayoría de los estudiantes es buena, por lo tanto, es necesario desarrollar actividades en cllos que les ayude a influir en pro de modos y estilos de vida favorables, de manera que éstas ayuden a rescatar una correcta higiene dental.

En cuanto a la Práctica se concluve que la mayoría de los estudiantes al utilizar los diferentes aditamentos de higiene bucal se autocalifican con mejores hábitos de higiene bucal. Por tanto, el compromiso preventivo con esta población de jóvenes universitarios debe estar encaminado principalmente a explicar la forma y frecuencia del cepillado, beneficios del uso de la seda y el enjuague bucal, la importancia de las visitas periódicas al odontólogo, ya que estos aspectos son importantes que los conozcan, para que se sientan motivados a principiar acciones encaminadas al cuido y mantenimiento de la salud bucal, la implementación de estas acciones a través de un programa, redundará en una disminución efectiva de las enfermedades bucales, lo cual puede ser comprobado con cl paso del tiempo al incorporar una acción de seguimiento.

La mayoria de la población adulta joven participante de este estudio padece de algún problema bucal, entre la que sobresale la caries dental, por lo que se puede inferir de que en ellos existe ausencia de una cultura de prevención. Por lo tanto es necesario, establecer en ellos no solo acciones de prevención si que tambien de intervención, de modo que recuperen por completo la salud bucal.

Orozco y Jiménez mencionan que es recomendable que las actividades preventivas sc realicen en la etapa preescolar o antes si fuese posible. En este sentido, la responsabilidad que debe asumir el gremio odontológico y las escuelas formadoras de odontólogos es decisiva, ya que es necesario realizar esfuerzos en conjunto, tal como to menciona Armenteros, Hernández y Olalde para realizar una intervención dirigida a diferentes grupos de población desde las edades preescolares para la prevención y promoción de la salud, en diferentes centros educacionales y al conjunto de la comunidad; de esta manera será posible la transmisión de conocimientos y el desarrollo de conductas encaminadas a la salud bucal.

\section{Recomendaciones}

Promover la formación de equipos multidisciplinarios constituidos por profesionales de las facultades, tanto del área de la salud como de educación, con el objetivo de enriquecer conocimientos, actitudes y prácticas sobre el mantenimiento de la salud integral de toda la comunidad universitaria.

A las facultades de Odontología:

- Realizar una investigación sobre la misma temática dentro de sus Facultades con el objetivo de conocer el nivel de conocimiento, actitudes y prácticas de todos sus integrantes.

- Que al interior de las Instituciones de Educación superior que cuentan con esta facultad adquieran un papel protagónico en la promoción de la salud bucal.

- Implementar un programa de promoción de la salud bucal que incluya la educación en todas las áreas clinicas de la facultad.

- Involucrar a los estudiantes para implementar un programa de prevención dentro y fuera de la Universidad para poner en práctica los conocimientos teóricos adquiridos durante la carrera.

A las personas en general:

- Enriquecer más sus conocimientos sobre el mantenimiento de la salud y sus consecuencias.

- Solicitar al Odontólogo la instrucción respecto a la elección de los aditamentos necesarios para mantener la higiene bucal de acuerdo a su propia necesidad.

- Practicar los hábitos de higiene bucal recomendados a fin de evitar las consecuencias 


\section{Fuentes de Información Consultadas}

1. Armenteros fimines, Santa; Hernandez. Fiménez María de los Angeles y Olalde fover, Luisa. Estrategia para aumeniar la higiene bucal en Los adolescantes. Publicado: $12 / 5 / 2007$ |Odontologia y Estomatología, Medicina Precientiva y Salud Publica. hitp:/ / waro portalesmedicos.com/publicaciones/arlicles/837/1/ Estrategia para-aumentar-la-higiene-bucal-en-los-adolescentes. html Fecha de acceso 30 de julio de 2007.

2. Avendaño Valiente, Adrián; Artiga, Yesenza. Métodos hiviénicos orales de la población en general. Estadísticas periodontal de paicntes atendidos en las clinicas.

htp://zowew.usam edusv/ICarticulos.php. Fecha de acceso el 09 de Agosto de 2005

3. Avendaño Valiente, Adrián.

http://zezus periodoncia.conso/periodonial. Fecha de accesa 07 de julio de 2005

4. Brenes, William; Sosa, Doris, Epidemiologia bucal y accesibilidad a los servicios odontológicos de un grupo de adolescentes. Rez. Cost. Cienc. Med. 1986; 7(4):311-314.

5. hitp://www binasss.sa.cr/adolescencia/epidemialogiahtm. Fecha de acceso 23 de jutio de 2007.

6. Consumeres EROSh7. Una mala huiene bucal provoca que el $70 \%$ de los españoles sufra sangrado de encias, según un estudio. htip://urewe.conswmer.es/web/es/salud/2005/12/29/148179. php?print=lrue Fecha de acceso 23 de julio de 2007.

7. Diaz del Mazo L, Pórez Núñez HM, Garcia Diaz $R$ de la $C$, $O^{\prime}$ Connor Martinez I Instruction "Sonnisas Saludables" para capacilar a adolescentes sobre salud bucal farticulo en linea]. MEDISAN 2003:7(4).

hitp://brs.sld.cu/reristas/san/vol7_t_03/san 1 1403.htm Fonsulta: 31 de Mavo de 20057.

8. DIGESTIC. Encuesta de Hogares de Propósitos Mülizbles 2004. htp:/ / wowedigeslyc.gob.sv/publicaiones/EHPM2004/2004/I NDICADORESHOGARES/depto/departamento2004.pilf Fecha de Acceso I 4 de julio de 2005.

9. DIGESTTC. Encuesta de Hogares de Propásilos Múltiples 2004. hup://wwadigestyc goh.so/publicaciones/EHPM2004/2004/P PALESRESULTADOS/principalesresultados2004.pulf Fecha de Acreso 14 de jutio de 2005.

10. Garcia Bocaranda, Blanca. Desnutrición materna perjudica dentición del bebé.

hltp://www. gentiuno. comarticulo, asp?arliculo $=1.593$ Publicado el 31 de Agosto de 2004. Focha de acceso 01 de junio de 200.5.

11. Kalz. Simon; at al. Odontologia preventiva en acción. $3^{\circ}$ edición. Edilorial Médica Panamerirana. 1982. Buenos Aires, Argentina.

12. Kuska, Bob Study. Finds Periodontal Treatment Does Nol Lower Preterm Birth Risk

http://wawe:midernih gov/News Releases/PeriodontalPrelermBirth Rist.htm Fecha de acceso 23 de jutio de 2007

13. López Vasquez, Sandra Lizeth; Martinez Baires, Deisy Auda; Martinez González, Claudia Carolina; Rivas Pérez, Luisa Jeannette. "Estudio de la prevalencia de lesiones cariosas en la población adulia de ambos géneros comprendido entre las edades de 20 a 59 años, en las unidades de Monte San funn Candelaria, San ramón del Departamento de Cuscatlán en el periodo 2002 " Iniversidad de El Salvador. Ciudad Uninersilana, Septiombre de 2003. Fecha de acceso 28 de junio de 200.5.

14. Ministerio de Salud Pública y Asistencia Social. Investigación epulemiológica de caries y Fluorosis Dental.

hup://uzeretmspas.gob.su/i_salud4_01.asp. Fecha de acceso 31 de Mave de 2005 .

15. Ministerio de Satud Pública y Asistencia Social. Programa Nacional de Odontologia. http:l/www.mspas.gab.so/p_saladbucal.asp Fecha de acceso 23 de jutio de 2007.

16. Ministerio de Salud Pública y Asistencia Social. Programa Esinela Saludable 1995-2003.

htlp:/ /woure.mspas.gob.sv/p_esc_saludable.asp Fecha de acceso 2.3 de julio de 2007.

17. National Institute of dental and Craneofacial Reseawch NIDCR. Oral Systemic Health Connection. Updated March 29, 2007 http://wrere:nidcrnih. gov/HealthInformation/DiseaserAndConditi ons/OralSystemicHeal__thConnection/default. htm Fecha de acceso 23 de julio de 2007
18. OMS. Informe sobre la salud en el mundo. Indicadores básicos de todos los estados miembros.

hitp:/ wow who.inl/wh/2004/annex/lopic/en/annex_I_es.pfd Fecha de acceso 37 de Mavo de 2005.

19. Obsernatorio Nacional de la Salud Bucal y la Práctica Odontalógica hutp:I onsb.udectedu.co Fecha de accesn 2.3 de julio de 2007.

20. Oral Health Spanish, Salud Bucal Asociación Dental de Califormia. Salud Bucal y Enfermedades Sislémicas. AVANZA VDO. JUVTOS. http://wwatcda.org/popup/Oral__Heatth_Spanish Fecha de acceso 23 de julio de 2007.

21. Orozco, Vancy Eliobeth; Zurita, Guana Jiménez et al. Estadio de salud bucodenlal en estudiantes de la carrera de cimijano dentista de la FES Iztacala. Revista ADM, Vol. LXIV. No. 2 Marzo-Abril 2007 pp 52-55. Fecha de aceeso 23 de actubre de 2007

22. Otero Martinez. Jorge; Toledo Reyes, Izlian. Situación de salud bucal en la población adulta (35-59 años). Villa Clara. Cuba. 2006hth://wewe,porlalesmedicos,com/publicaciones/articles/546 11/Situacion-de-salud-bucal-en-ta-poblacion-adulat-3.5-59-anas Villa-Clara-Cuba-2006.html Fecha de acceso 23 de julio de 2007.

23.Petersen P. The World Oral Healh Report 2003. Continuos improvement of oral health in the 21 st century - the approach of the WHO Global Oral Healih Programme.

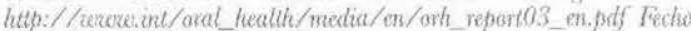
de acceso 07 de junio de 2005

24.Revista Virtual ODONTOLOGLA CLINTCA. Prevención de las enfermedades dentales y bucales. hitp:/ / werwodontomarketing com/odontologiabravenciondefinicion.htm. Fecha de arceso 23 de julio de 2007.

25. Rodriguez Galzadilia, Amado; Delgado Méndez, Luis. Diagnóstico de salud bucal. Rev Cubana Estomatol 1996:33(1) hutp://bess.std.cu/revistas/est/iol.33_1_96/est01196.htm Fecha de acreso 23 de jutio de 2007.

26.Romero Diaz, Manuel H; Gueorguieza, Marieta Petkova; Mánquez, Ricardo M. Romero. Enfermedades bucales con Repercusión Sistémica. hutp://sisbib.unmsm.edu.pe/BVRevistas/adontologia/volB NT/a 10.thm Fecha de acceso 01 de junio de 2005.

27.Ruiz Hernández, Mónica; Reyes, Jesús Antonio, Bayona Rolando. Proyecto "El Club de la Sonrisa Feliz"

hth: / / iwou. fepafem.orgeve/investigaciones/amo2002/sonviza fel z.htm. Fecha de arceso 31 de Mayo de 2005.

28. Sosa, Lucio Manuel: et al. Ievantamiento epulemiológico bucal en escolares de $1^{\circ}$ y $2^{\circ}$ etapa. Ahril 2003. Sintesis: Tesis de Grado presentado en Junio de 2.003. Hospital Vuctorino Santaella Ruiz. http:/ /rwwe odontologia-online com/casos/bart/LST/LSTOS/ lst08.html Fecha de acceso 23 de julio de 2007

29. Suárez Morales, Xiomara: Pombo, Ada Broche; et al. Análisis de la situación de salud de 20 fomilias perienecientes al policlinico XX aniversario. 2003 Http://www.odontologia online.com/casos/parl/ $x s \mathrm{~m} / \mathrm{xsm01} / \mathrm{xsm01}$. html

30. Situación de Salud Bucal y Determinantes. Observatono. Nacional de la Salud Bucal y la Práctica Odontológica.

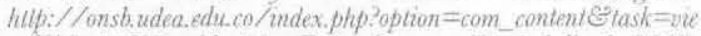
woid=13 Eltemid = 11 Fecha de accesa 23 de julio de 2007.

31. Sheiham. Autre). Declaracioin de Berlin para la Saludy Servicios Odontológicos. Un paso adelante para la cooperación mundial. Departamento de Epidemiologia y Salud Publina, Liniversily College London Medical School, Ingiaterra.

httb://wrwatibiblio org/tafi/cedros/espanol/nezesletter/n.5/Berhah htm! Fecho de acceso 23 de jubio de 2007.

32. Tascon, Jorge Eduardo: Cabrera, Gistavo Alonso. Creencias sobre caries e higiene oral en adolescentes del Valle del Cauca. Colomb. Med. conlinel. jun. 2005, vol.36, no.2 reitado 19 Julio 2007], p.73-78. Disponible en la World Wide Web:

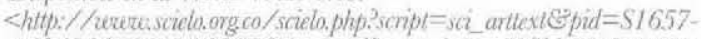

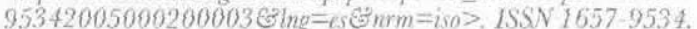
Fecha de acreso 19 de juito de 2007

33. Aumenta la incidencin de enformedades bucodentales en los paises pobres. hltp://wrews.geodental.net/article_7563.html Publicarion 174 de Geodental - Ouintersence. Publicada el Miénoles 06 de Octubre del 2004 . Fecha de acceso el 07 de junio de 2005.

3f. Wevant Rj, Manz M. Corby P. Rustzeld L, Clase 7. Factors associated with parents' and adolescents' perceptions of oral health and need for denlal treatment. Community Dent Oral Epidemiol 2007; 35: $321-330$. 